\title{
Compact Patch Antenna with Vertical Polarization and Omni-Directional Radiation Characteristics
}

\author{
Chun Xu Mao, Member, IEEE, Mohsen Khalily, Senior Member, IEEE, Long Zhang, Member, IEEE, \\ Pei Xiao, Senior Member, IEEE, Yuhang Sun, and Douglas H. Werner, Fellow, IEEE
}

\begin{abstract}
This communication proposes a compact, low-profile patch antenna with omni-directional radiation pattern and vertical polarization. A pair of shorted patches are excited in-phase to achieve the omni-directivity and the vertical polarization, simultaneously. The principle is to excite two back-to-back arranged shorted patches to generate symmetrical electric field (E-field) distributions normal to the ground plane. Analytical study on how to generate the omni-directional radiation pattern is carried out. Base on this study, we found the spacing in-between the two patches have little influence on the radiation characteristics, which provides another flexibility in the design. In addition, the shape of the patch and the corresponding field distribution are investigated to further improve the omni-directivity. To improve the impedance bandwidth, resonant structures are inserted in-between the patches, producing the $2^{\text {nd }}$ order response in frequency. The antenna has been fabricated and characterized, and the measured results are in a reasonable agreement with the simulations, showing that the proposed antenna is suitable for potential surface-mount wireless applications.
\end{abstract}

Index Terms - antenna, compact, low-profile, vertical polarization, omni-directional.

\section{INTRODUCTION}

Antenna with omni-directional radiation characteristics in the azimuthal plane has shown many advantages in short-distance wireless applications, such as intelligent transportation system (ITS), wireless broadcasting, and wearable devices. An omni-directional antenna can ensure a steady connection with other signal sources around when the positions and orientations of the receiving and transmitting antennas are altered [1]-[2]. Traditionally, omni-directivity can be achieved by using a dipole or a monopole. However, these approaches suffer from high profile, and thus, they are not suitable for portable devices, especially the surface mount devices.

Omni-directional antennas with both circular polarization (CP) and linear polarization (LP) characteristics were intensively explored in the past years. In [3]-[6], the $\mathrm{TM}_{10}$ and $\mathrm{TM}_{20}$-mode of the patch are excited to generate the vertical polarization and circular strips or slots to generate the horizontal polarization in the azimuthal plane, resulting in CP radiation. Omni-directional antenna with LP radiation in vertical plane can also find potential applications in internet of thing (IoT) and on-body wearable applications [7]-[8], etc. Various linearly polarized

This work was supported by UK EPSRC project "MILLIBAN" under Grant EP/P008402/2. The work was also supported by the National Science Foundation ASSIST Nanosystems ERC under Award EEC-1160483. (Corresponding author: Chunxu Mao).

C. Mao, M. Khalily, and P. Xiao are with Institute for Communication Systems (ICS), 5G Innovation Centre (5GIC), University of Surrey, Guildford, GU2 7XH, UK (e-mail: c.mao@surrey.ac.uk).

L. Zhang and Y. Sun are with the College of Electronics and Information Engineering, Shenzhen University, Shenzhen 518060, China.

D. Werner is with the Electrical Engineering Department, The Pennsylvania State University, University Park, PA 16802 USA.

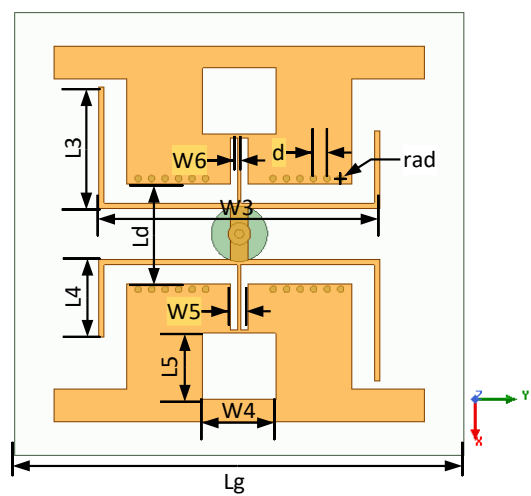

(a)

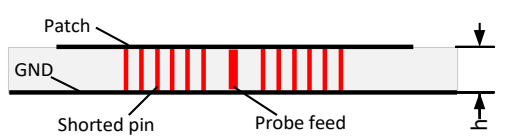

(b)

Fig. 1. Configurations of the proposed omni-directional antenna, (a) top view, (b) side view. $L 1=40 \mathrm{~mm}, L 2=3 \mathrm{~mm}, L 3=10.5 \mathrm{~mm}, L 4=4.5 \mathrm{~mm}$, $L 5=6 \mathrm{~mm}, L 6=8 \mathrm{~mm}, W 1=40 \mathrm{~mm}, W 2=33 \mathrm{~mm}, W 3=25 \mathrm{~mm}, W 4=6.5$ $\mathrm{mm}, W 5=1.5 \mathrm{~mm}, W 6=0.4 \mathrm{~mm}, d=1.2 \mathrm{~mm}, \mathrm{rad}=0.3 \mathrm{~mm}$.

omni-directional antennas with broad bandwidth have been reported in [9]-[17]. A common way is to excite a symmetrical patch at the center and load the antenna with capacitive plate and shorted pins. Although good performance can be achieved, these antennas have up-tilt radiation pattern due to the excitation of the higher modes.

In this communication, a new method of realizing the LP omnidirectional patch antenna is proposed by employing two back-to-back arranged shorted patches. Such a layout can generate symmetrical electric field distribution, and therefore, omni-directional radiation pattern in the azimuth plane. An analytical study is first carried out to illustrate the principles of producing the vertical polarization and the omni-directional pattern in the far-field. The technical contribution of this work includes: (1) a distributed method to design vertically polarized omni-directional patch antenna by exciting a pair of back-to-back arranged shorted patch; (2) analytical study and experimental validation of the proposed antenna; (3) investigation of the methods of improving the omni-directivity by modifying the shape of the patch and the corresponding current distribution; (4) a new method of enhancing the bandwidth by introducing two strip lines between the two patches without increasing antenna's thickness. Moreover, the antenna performance is investigated when it is placed on a large copper plane and human phantom, respectively.

\section{Antenna Design}

In this section, a low-profile, vertically polarized planar antenna with omni-directional radiation pattern is presented and studied. First, 


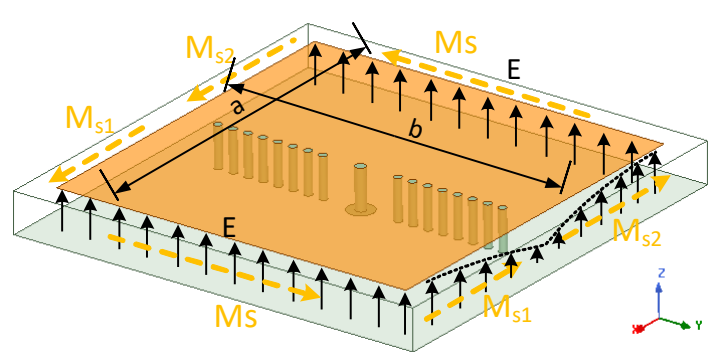

(a)

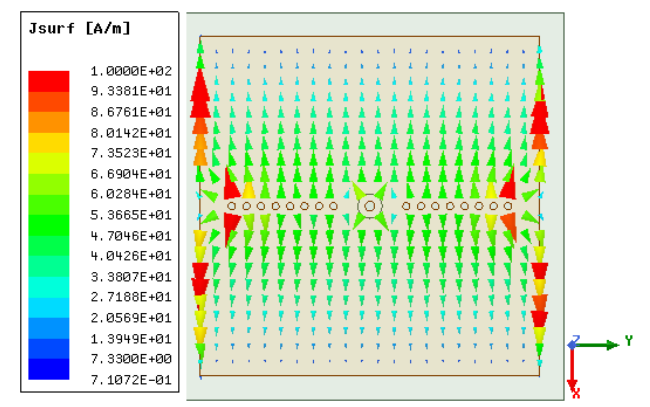

(b)

Fig. 2. (a) Configuration of simplified shorted patch antenna (Type-I) and its E-field distribution, (b) simulated current distribution at $2.45 \mathrm{GHz}$.

the approaches of achieving the omni-directional radiation pattern and vertical polarization are studied. Then, studies of how to improve the omni-directivity of the pattern and the bandwidth are carried out.

\section{A. Configurations}

Fig. 1 shows the configuration of the proposed omni-directional antenna. The antenna has a single substrate with a ground plane on the bottom layer. On the top layer, two T-shaped patches are placed back-to-back and fed by a probe at the center. Two rows of metallic pins are inserted to short the patches to the ground plane on the bottom. The length from the opened end to the shorted end of the patch is approximately a $\lambda / 4$ at the center frequency $(2.45 \mathrm{GHz})$. Two strip lines are inserted in-between the patches to enhance the impedance bandwidth of the antenna, which will be detailed in part-C. The antenna is implemented on a RO4003C substrate with a dimension of $40 \mathrm{~mm} \times 40 \mathrm{~mm}$, which is corresponding to $0.33 \lambda \times 0.33 \lambda$ at 2.45 GHz. The relative permittivity and the loss tangent of the substrate are 3.55 and 0.0027 , respectively. The antenna design and optimization were performed using the High Frequency Structural Simulator (HFSS) and the optimized parameters are listed in the caption of Fig. 1.

\section{B. Omni-Directional Radiation}

To illustrate the principles of achieving the omni-directional radiation, the configuration in Fig. 1 is simplified by removing the two strip lines and combining the two patches together (denoted as Type-I), as shown in Fig. 2(a). The introduced metallic vias divide the patch into two identical sections and change the electric-field distribution characteristics, and therefore the far-field pattern. Due to the symmetry, the patch has uniform electric field distribution $\boldsymbol{E}$ on the two opening slots with a direction from ground to the patch (or reverse), as plotted in Fig. 2. These two opening slots can be equivalent as two magnetic current in opposite directions (+/-y axis), denoted as $\boldsymbol{M s}$. Viewing the electric field distribution on the lateral sides, we can observe that the direction of the E-field is the same as that of the two opening slots, but the intensity is gradually reduced from both ends to the center, following cosine function. Fig. 2(b) shows the simulated current distribution on the patch at $2.45 \mathrm{GHz}$. The

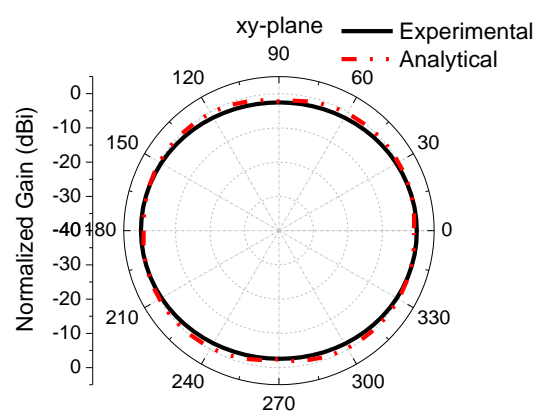

Fig. 3. Comparison of the analytical and experimental normalized H-plane radiation patterns of the Type-I antenna.

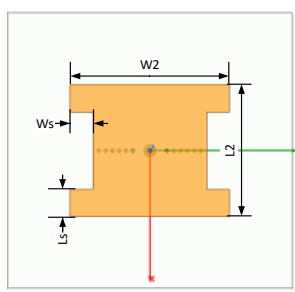

(a)

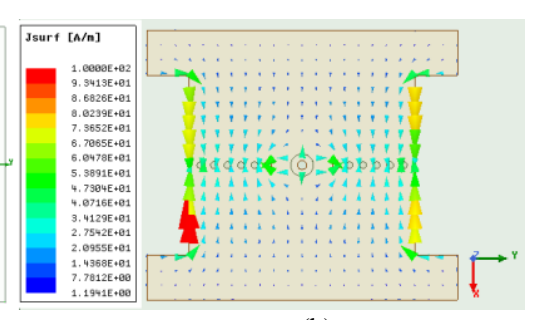

(b)
Fig. 4. (a) Configuration of the Type-II antenna, (b) simulated current distribution at $2.45 \mathrm{GHz}$.

current flows from the shorted pins in the middle to both ends of the patch and is symmetrical to the pins.

Different from traditional patch antenna that only two slots contribute to the radiation [18], the far-field radiation of the proposed antenna is attributed by all four slots with uniform electric-field direction in the z-axis direction. Such an E-field distribution can be equivalent to a magnetic current loop in xy-plane, as also shown in Fig. 2(b). It is well known that a magnetic current loop in horizontal plane will result in vertical polarization radiation $\left(E_{\theta}\right)$ in the far-field. The far-field E-field distribution intensity of the proposed antenna can be derived by calculating the radiations of the four magnetic currents. According to the methods of calculating the radiation of a rectangular radiating slot [18], the far-field E-field of one opening slot in y-axis direction $(\mathrm{x}=+\mathrm{a} / 2)$ can be derived,

$$
\begin{aligned}
E_{\theta 1}=- & \frac{j k b h E e^{-j k r}}{4 \pi r}\left(\cos \phi \cdot \frac{\sin (X)}{X} \cdot \frac{\sin (Y)}{Y}\right) \\
& \left\{\begin{array}{l}
X=\frac{k b \sin \theta \cdot \sin \phi}{2} \\
Y=\frac{k h \cos \theta}{2}
\end{array}\right.
\end{aligned}
$$

where, $k$ is the wavenumber at the designed frequency, $h$ is the height of the substrate, $E$ is the electric field intensity. When $\theta=\pi / 2$ is applied to (1)-(2), the E-field distribution in the H-plane can be derived,

$$
E_{\theta 1_{-} H}=-\frac{j k b h E}{4 \pi r} e^{-j k r} \cdot\left[\cos \phi \frac{\sin \left(\frac{k b \sin \phi}{2}\right)}{\frac{k b \sin \phi}{2}}\right]
$$

Similarly, the E-field of the other three radiating slots can be derived. It should be noted that the E-field intensity of the two-lateral radiating slots is weaker than the other opening slots. To reduce the analytical complexity, the E-filed intensity of the lateral slots is approximated to $1 / \sqrt{2}$ of that in the opening slots. Then, the combined E-field of the four radiating slots in the H-plane can be calculated and plotted in Fig. 3. As can be seen, the experimental pattern agrees well with the analytical result, showing a good omni-directionality in the H-plane 


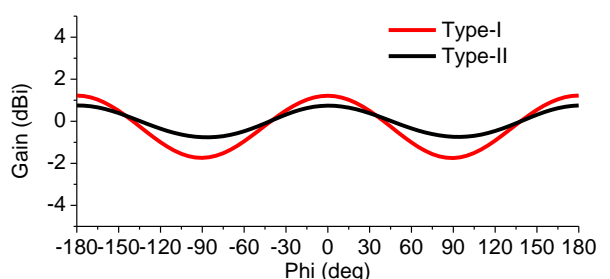

Fig. 5. Comparison of the simulated H-plane radiation patterns between Type-I and Type-II antennas.

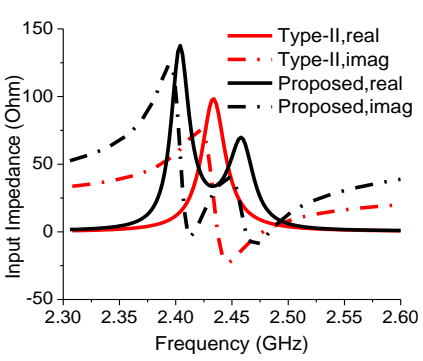

(a)

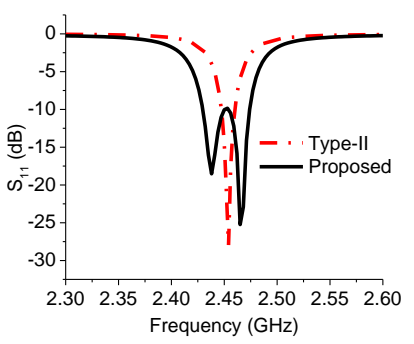

(b)
Fig. 6. Comparisons between the Type-II and the proposed antennas: (a) input impedance, and (b) $S_{11}$.

(xy-plane). Due to a weaker E-field distribution along the two lateral radiating slots (normal to $\mathrm{y}$-axis), the radiation in $\varphi= \pm 90$ degrees are about $3 \mathrm{~dB}$ lower than that in $\varphi=0$ (180) degrees.

In order to improve the omni-directional performance, the shape of the patch of Type-I antenna is modified by adding four stubs $(L s \times$ $W s$ ), as shown in Fig. 4(a). The simulated current distribution is presented in Fig. 4(b). Compared with Fig. 2(b), the extended stubs change the current distribution on the patch, generating a current component in $+/$-y directions. This additional component can enhance the radiation in $\varphi= \pm 90$ degrees, producing an improved omni-directivity. It is observed from Fig. 4(b) that the length of the path from the open end of the stub to the shorted end $\left(L_{2} / 2+W s\right)$ is about a quarter wavelength. Fig. 5 compares the simulated H-plane radiation patterns between Type-I and Type-II antennas. It is observed the radiation fluctuation is reduced from $3 \mathrm{~dB}$ for the Type-I antenna to about $1.5 \mathrm{~dB}$ for the Type-II antenna.

\section{Impedance Bandwidth Improvement}

Another issue to be concerned is the bandwidth. To enhance it, the patch is split into two parts and two strip lines are inserted in between them, as shown in Fig. 1. This structural modification does not change the field distribution and thus the radiation can be kept. The strip lines serve as $\lambda / 2$ microstrip resonator, i.e. the total length of $L_{3}+L_{4}+W_{3} \approx$ $\lambda \mathrm{g} / 2$. The strip lines are served as the first stage of the resonant circuit, whereas the shorted patches serve as the radiator and the last resonator. Fig. 6 compares input impedance and $S_{11}$ of the antennas with and without the strip lines. As observed, the proposed antenna has two resonances at around 2.4 and $2.46 \mathrm{GHz}$, forming a second-order resonant characteristic [19]-[20]. Fig. 6(b) compares the impedance bandwidths between the Type-II and proposed antenna. It is observed that Type-II antenna has a bandwidth of only $15 \mathrm{MHz}$, which is enhanced to over $50 \mathrm{MHz}$ for the proposed antenna.

\section{RESUlTS AND DisCUSSION}

The proposed antenna was fabricated and the prototype is shown in Fig. 7. Fig. 8 shows the simulated and measured S-parameters and the gains of the proposed antenna. The measured results concur with the simulations, exhibiting a $-10 \mathrm{~dB}$ bandwidth from 2.43 to $2.49 \mathrm{GHz}$. The measured bandwidth is slightly wider than the simulation result, which is attributed to the insertion loss of the SMA connector. The

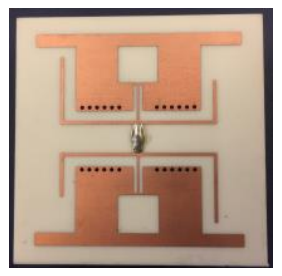

(a)

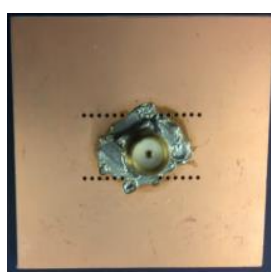

(b)
Fig. 7. Fabricated antenna prototype, (a) top view, (b) bottom view.

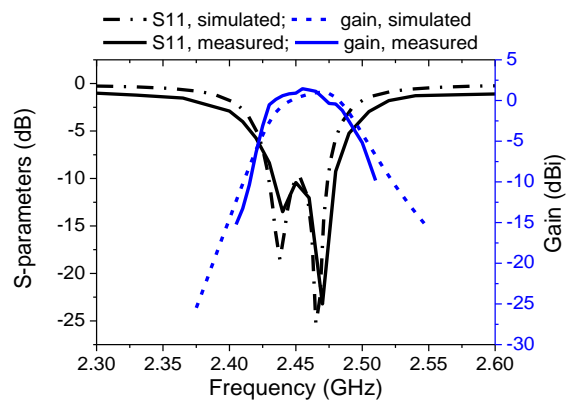

Fig. 8. Simulated and measured S-parameters and gains of the proposed omni-directional antenna.

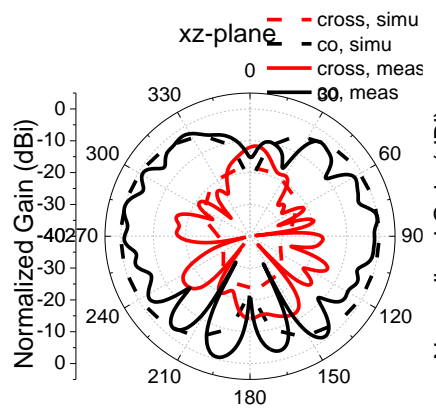

(a)

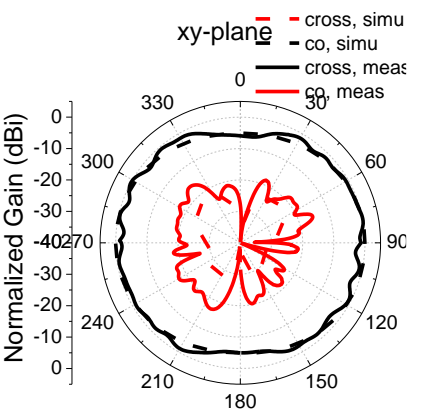

(b)
Fig. 9. Simulated and measured normalized radiation patterns of the omni-directional antenna at $2.45 \mathrm{GHz}$ : (a) xz-plane (E-plane), (b) xy-plane (H-plane).

measured gains are about $1 \mathrm{dBi}$ in the band of interest and agree well with the simulations.

Fig. 9 shows the simulated and measured normalized radiation patterns at $2.45 \mathrm{GHz}$. The antenna exhibits a dumbbell-like radiation pattern in the E-plane and omni-directional pattern in the H-plane. The measurements agree reasonably well with the simulations but exhibiting some ripples on the patterns. This is mainly caused by the flowing current outside the cable due to a small ground plane, which is superposed to the main radiation, leading to unexpected radiation nulls. This can be solved by increasing the ground size or adding a sleeve balun on the cable.

\section{Potential Application Study}

\section{A. On Conducting Plate}

One of the potential applications of the proposed antenna is in surface-mount platform, e.g. on a vehicle. Thus, is interesting to study the performance of the antenna when the size of the ground plane is enlarged. To this end, the antenna is placed on a copper plate with a dimension of $L_{\text {copper }}$, as shown in Fig. 10(a). Fig. 10(b) shows the simulated E-plane radiation pattern with different sizes of the copper plate. As observed, the antenna has the maximum radiation in $\theta=90$ degrees when the size of copper plate is small. The beam is gradually tilt up to $\theta=45$ degrees when $L_{\text {copper }}$ is increased to $140 \mathrm{~mm}$ due to the reflection function of the copper plate. 


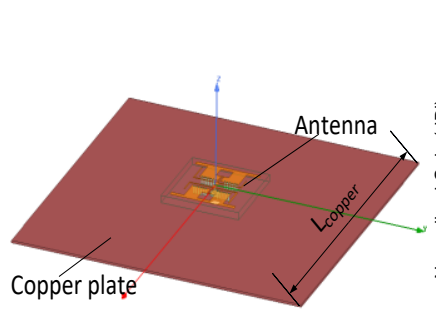

(a)

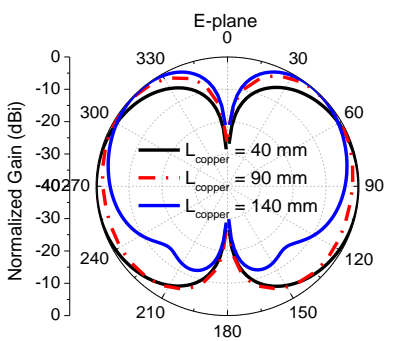

(b)
Fig. 10. (a) Layout of the proposed antenna on a rectangular copper plate, (b) Simulated E-plane radiation pattern with different sizes of copper plate.

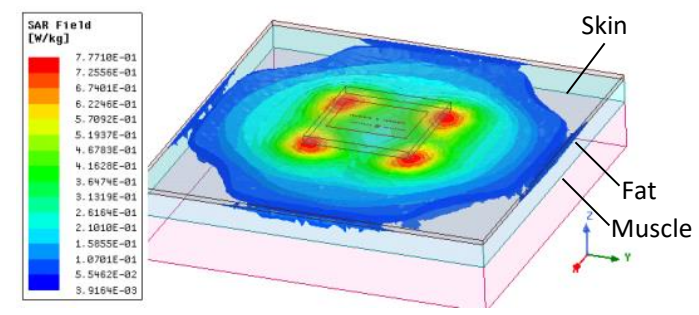

(a)

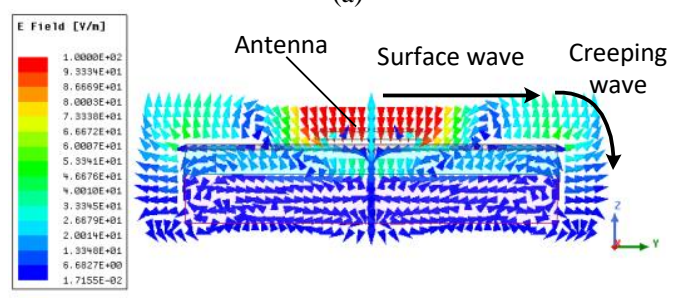

(b)

Fig. 11. (a) Simulated $1 \mathrm{~g}$ averaged SAR value in the tissue, and (b) side view of the simulated electric-field distribution.

\section{B. On Human Phantom}

Another potential application of the proposed antenna is in wearable systems where a low-profile antenna with linear polarization normal to the surface of human phantom is desired to excite surface wave. In addition, a low specific absorption rate (SAR) is required to reduce the absorption by human tissue. For evaluation, a human phantom composed of skin layer, fat layer, and muscle layer is modeled, as shown in Fig. 11(a). The phantom has a larger size than the antenna and the thicknesses are $2 \mathrm{~mm}, 5 \mathrm{~mm}$, and $15 \mathrm{~mm}$ for the skin, fat, and muscle layers, respectively. The simulated maximum averaged SAR in the phantom is $0.78 \mathrm{~W} / \mathrm{kg}$ when the incident power is $100 \mathrm{~mW}$, which is far below the safety value of $1.6 \mathrm{~W} / \mathrm{kg}$. As shown by Fig. 11(b), a strong surface wave can be generated on the interface between the phantom and the air, producing creeping wave at the edge of the phantom for improving the link non-line-of-sight.

\section{CONCLUSION}

In this communication, a low-profile, LP omni-directional antenna with has been proposed for potential IoT and wearable applications. The omni-directionality and the LP in vertical plane have been achieved by exciting two physically separated shorted patches. The designing concept and approaches have been illustrated and verified experimentally. Such a distributed layout provides more flexibility in realizing the omni-directional antenna. The methods of improving omni-directivity and impedance bandwidth have been investigated. The predictions were validated by measurement results, demonstrating a good omni-directional performance, rendering it suitable for a wide range of wireless applications.

\section{REFERENCES}

[1] Y. Liu, X. Li, L. Yang, and Y. Liu, "A Dual-polarized dual-band antenna with omni-directional radiation patterns," IEEE Trans. Antennas Propag., vol. 65, no. 8, pp. 4259-4262, Aug. 2017.

[2] C. Mao, M. Khalily, P. Xiao, T. Brown, and S. Gao, "Planar sub-millimeter-wave array antenna with enhanced gain and reduced sidelobes for $5 \mathrm{G}$ broadcast applications," IEEE Trans. Antennas Propag., vol. 67, no. 1, pp. 160-168, Jan. 2019.

[3] B. C. Park, and J. H. Lee, "Omni-directional circularly polarized antenna utilizing zeroth-order resonance of epsilon negative transmission line," IEEE Trans. Antennas Propag., vol. 59, no. 7, pp. 2717-2721, Jul. 2011.

[4] Y. M. Cai, S. Gao, Y. Yin, W. L, and Q. Luo, "Compact-size low-profile wideband circularly polarized omnidirectional patch antenna with reconfigurable polarizations," IEEE Trans. Antennas Propag., vol. 64, no. 5, pp. 2016-2021, May 2011.

[5] Y. Yu, J. Xiong, and H. Li, "Compact omni-directional circularly polarised antenna utilising bended dipoles and integrated baluns," IET Microw. Antennas Propag., vol. 11, no. 10, pp. 1409-1414, Oct. 2017.

[6] W. Lin, and H. Wong, "Circularly polarized conical-beam antenna with wide bandwidth and low profile," IEEE Trans. Antennas Propag., vol. 62, no. 12, pp. 5974-5982, Dec. 2014.

[7] P. S. Hall et al., "Antennas and propagation for on-body communication systems," IEEE Antennas Propag. Mag., vol. 49, no. 3, pp. 41-58, Jun. 2007.

[8] D. H. Werner and Z. H. Jiang, "Electromagnetics of Body Area Networks: Antennas, Propagation, and RF Systems, Wiley-IEEE Press, 2016.

[9] H. Nakano, H. Iwaoka, K Morishita, and J. Yamauchi, "A wideband, low-profile, antenna composed of a conducting body of revolution and a shorted parasitic ring," IEEE Trans. Antennas Propag., vol. 56, no. 4, pp. 1187-1192, Apr. 2008.

[10] M. Koohestani, J. F. Zurcher, A. Moreira, and A. SKrivervik, "A novel low-profile, vertically polarized ultrawideband antenna for WBAN," IEEE Trans. Antennas Propag., vol. 62, no. 4, pp. 1888-1894, Apr. 2014.

[11] A. Elsherbini, and K. Sarabandi, "Very low-profile top-loaded UWB coupled sectorial loops antenna," IEEE Antennas Wireless Propag. Lett., vol. 10, pp. 800-803, 2011.

[12] J. Liu, Z. Tang, and Y. Yin, "Wideband omni-directional antenna with novel feeding structure for improving radiation patterns," Electron. Lett., vol. 53, pp. 830-832, 2017.

[13] D. Aten, and R. Haupt, "A wideband, low-profile, antenna composed of a conducting body of revolution and a shorted parasitic ring," IEEE Trans. Antennas Propag., vol. 60, no. 10, pp. 4485-4491, Oct. 2012.

[14] N. Behdad, M. Li and Y. Yusuf, "A very low-profile, omnidirectional, ultrawideband antenna," IEEE Antennas Wireless Propag. Lett., vol. 12, pp. 280-283, 2013.

[15] K. Ghaemi and N. Behdad, "A wideband, low-profile, antenna composed of a conducting body of revolution and a shorted parasitic ring," IEEE Trans. Antennas Propag., vol. 63, no. 8, pp. 3699-3705, Aug. 2015.

[16] N. N. Trong, A. Piotrowski, T. Kaufmann, and C. Fumeaux, "Low-profile wideband monopolar UHF antennas for integration onto vehicles and helmets," IEEE Trans. Antennas Propag., vol. 64, no. 6, pp. 2562-2568, Jun. 2016.

[17] F. Yang, Y. Rahmat-Samii, and A. Kishk, "Low-profile patch-fed surface wave antenna with a monopole-like radiation pattern," IET Microw. Antennas Propag., vol. 1, no. 1, pp. 261-266, Jan. 2007.

[18] C. A. Balanis, Antenna Theory, Analysis and Design, Hoboken, NJ, USA: Wiley, 2005.

[19] C. X. Mao, S. Gao, and Y. Wang, "Highly integrated full-duplex Tx/Rx antennas for vehicular communications," IEEE Trans. Veh. Technol., vol. 67, no. 5, pp. 4059-4070, 2018.

[20] C. X. Mao, S. Gao, Y. Wang, Q. Luo, and Q. Chu, "A shared-aperture dual-band dual-polarized filtering-antenna-array with improved frequency response," IEEE Trans. Antennas Propag., vol. 65, no. 4, pp. 1836-1844, Apr. 2017. 